\title{
Variabilidad espacio-temporal de las comunidades de plantas acuáticas en un humedal de los Llanos de Venezuela
}

\author{
Anabel Rial B. \\ Museo de Historia Natural La Salle, Apdo. 1930 - Caracas 1010-A, Venezuela; anabel.rial@fundacionlasalle.org.ve \\ Recibido 22-XI-2002. Corregido 28-X-2003. Aceptado 15-VI-2004.

\begin{abstract}
Spatial-Temporal variability of aquatic plant communities in the Venezuelan Llanos. The richness and abundance of aquatic plant communities were studied in 14 habitats of the Venezuelan "llanos" $\left(07^{\circ} 35^{\text {'- }}\right.$ $07^{\circ} 55^{\prime} \mathrm{N}-68^{\circ} 50^{\prime}-69^{\circ} 00^{\prime} \mathrm{W}$, Apure) during an annual cycle. Annual means were $27^{\circ} \mathrm{C}, 115 \mathrm{~mm}$ rainfall and $77 \%$ relative humidity. A permanent transect was set in each habitat (ten consecutive square meter quadrats from the shore to the water). The plants and the area they covered in each quadrat were recorded monthly for a year. The total richness was 69 species. Alatalo and Alatalo's diversity analysis indicats that season, and its correlate, water level, influence species diversity and abundance more than habitat (spatial factor: geometric index-Euclidean distance). Rev. Biol. Trop. 54(2): 403-413. Epub 2006 Jun 01.
\end{abstract}

Key words: aquatic plants, diversity, hydroperiod, wetland, Orinoco floodplain, Llanos, Venezuela.

Los humedales suramericanos están controlados por el pulso de inundación (Neiff 2001). La variación en el nivel del agua es el factor que influye en mayor medida sobre la riqueza y abundancia de las comunidades vegetales acuáticas en la planicie inundable de los Llanos de Apure (Rial 2001). Las características de cada ambiente o tipo de hábitat (morfología de la cubeta, gradiente de profundidad, tipo de fondo, propiedades fisicoquímicas del agua, entre otras) ejercen menor influencia sobre dichos parámetros. Las comunidades se definen aquí como conjuntos de plantas de diferentes especies y formas de crecimiento, que habitan juntas y asociadas a un cuerpo de agua determinado durante un tiempo variable del ciclo anual. Se refiere a unidades de carácter práctico; segmentos relativamente uniformes del ecosistema que resultan de la técnica de muestreo (Margalef 1974).

Los estudios ecológicos en humedales de Suramérica han tenido especial atención en las últimas décadas (Neiff 1978, 1986, 2001, Beck 1984, Junk 1984,1993, Mereles y
Kochalca 1992, Gantes y Tur 1993, Werhoven y Peters 1993, Colonnello 1995, Gordon 2000, Rial 2000, 2001), entre otros. En los Llanos inundables de Apure, se estudió mensualmente y durante un ciclo hidrológico, un conjunto de hábitat que representa las zonas más dinámicas del humedal (zonas de transición-ecotonos), con el fin de determinar si las mayores variaciones se debían a la fluctuación en el nivel del agua y cuales serían los efectos de las combinaciones entre variación del tipo de ambiente y estacionalidad. El objetivo del trabajo fue investigar sistemáticamente los cambios de las comunidades vegetales acuáticas -en términos de su riqueza y abundancia- en diversos ambientes durante los doce meses del ciclo hidrológico. Asimismo, cuantificar y describir la intensidad de dichas variaciones durante los periodos críticos del ciclo hidrológico (especialmente fin de la sequía e inicio de lluvias). Finalmente, precisar las diferencias que observamos cualitativamente y a priori, respecto a cuanto más diferente puede ser la comunidad de un mismo ambiente en los meses de 
transición lluvia-sequía, al compararla con la de otro ambiente durante la misma época.

Area de estudio. El estudio se llevo a cabo en el Hato El Frío, una extensión de 80.000 ha de sabanas inundables, situado entre las coordenadas geográficas $07^{\circ} 35^{\prime}-07^{\circ} 55^{\prime} \mathrm{N}-68^{\circ} 50^{\prime}$ $69^{\circ} 00^{\prime}$ 'W en el Estado Apure, al suroeste de Venezuela. Es una región de clima cálido con promedios mensuales de temperatura de $27^{\circ} \mathrm{C}$, $115 \mathrm{~mm}$ de precipitación y $77 \%$ de humedad relativa (datos de la estación meteorológica de Mantecal). La biestacionalidad es marcada y apreciable en los periodos de lluvia y sequía que se inician en mayo y octubre, respectivamente. La fisiografía está caracterizada por los denominados bancos, bajíos y esteros, diferenciables fundamentalmente por su capacidad de retención de agua durante la sequía. En las zonas menos elevadas (esteros) se hallan diversos cuerpos de agua someros, polimícticos, lénticos y lóticos, permanentes y temporales tales como caños, esteros, bajíos, lagunas y préstamos (lagunas artificiales -abrevaderos para el ganado- derivadas de la extracción de tierra para construcción de terraplenes) cuyas profundidades máximas no superan los $2 \mathrm{~m}$. La planicie inundable del Hato el Frío alberga una riqueza superior a las 194 especies de plantas vasculares acuáticas (Rial 2001). Cabe resaltar su alta diversidad de aves, mamíferos, más de 180 especies de peces (Lasso 2000) y la presencia de reptiles en peligro de extinción como el caimán del Orinoco (Crocodilus intermedius).

\section{MATERIAL Y MÉTODOS}

Se estudiaron catorce ambientes representativos de los caños, esteros y lagunas del humedal llanero (Cuadro 1). Se dispuso en cada ambiente un transecto permanente, conformado por diez parcelas consecutivas de $1 \mathrm{~m}^{2}$ desde la orilla hasta el espejo de agua. Se

CUADRO 1

Transectos de estudio en los catorce ambientes representativos del humedal. Características fisicoquímicas registradas en el gradiente de profundidad durante el año 1997

Ámbito del parámetro en el gradiente durante el año 1997

\begin{tabular}{|c|c|c|c|c|c|c|}
\hline $\begin{array}{l}\text { Transecto } \\
\left(10 \mathrm{~m}^{2}\right)\end{array}$ & Ambiente & Tipo & profundidad & $\begin{array}{c}\mathrm{pH} \\
(\mathrm{cm})\end{array}$ & $\begin{array}{c}\mathrm{O} 2 \\
(\mathrm{mg} / \mathrm{l})\end{array}$ & $\begin{array}{l}\text { sólidos disueltos } \\
\quad(\mathrm{ppm})\end{array}$ \\
\hline R. Iguana I & estero & léntico & $10-115$ & $6.2-7.1$ & $0.2-6.7$ & $10-70$ \\
\hline R. Iguana II & estero & léntico & $0-140$ & $6.4-7.6$ & $0.2-13.6$ & $10-40$ \\
\hline Manirito & estero & léntico & $0-150$ & $6.3-7.9$ & $0.3-8.9$ & $20-170$ \\
\hline Morita I & estero & léntico & $0-109$ & $6.2-7.6$ & $0.2-11.1$ & $10-130$ \\
\hline Morita II & estero & léntico & $0-180$ & $6.8-7.8$ & $1-9.4$ & $10-70$ \\
\hline Boral I & laguna & léntico & $0-105$ & $5.5-7.6$ & $1.6-5.9$ & $10-60$ \\
\hline Boral II & laguna & léntico & $0-190$ & $5.7-7.4$ & $0.5-8.6$ & $10-510$ \\
\hline Mac. Caujaro & laguna & léntico & $0-88$ & $5.8-8.3$ & $0.3-17.9$ & $10-110$ \\
\hline Carmera & laguna & léntico & $0-114$ & $6.2-8.2$ & $1.1-8.7$ & $20-60$ \\
\hline Cochina & laguna & léntico & $0-92$ & $6.9-8$ & $2-13.2$ & $10-100$ \\
\hline Ventanas & laguna & léntico & $0-81$ & $6.4-8.2$ & $0.4-13.6$ & $30-700$ \\
\hline Préstamo & laguna & léntico & $0-200$ & $6.5-8.9$ & $0.7-8.6$ & $10-80$ \\
\hline Mucuritas & caño & lótico & $0-175$ & $6.2-8$ & $0.8-6.7$ & $10-60$ \\
\hline Macanillal & caño & lótico & $0-128$ & $6-8.1$ & $1.1-14.9$ & $10-30$ \\
\hline
\end{tabular}


determinó la frecuencia acumulada de especies mediante el muestreo sistemático y mensual de los catorce transectos. La abundancia en cada una de las diez parcelas de cada transecto, se estimó mediante la cobertura específica. Para tal fin se registraron intervalos de porcentaje correspondientes a un equivalente numérico del 1 al 6, según categorías modificadas de BraunBlanquet (1979).

La cobertura mensual por especie y transecto se obtuvo asumiendo que el valor máximo de una especie en cada parcela es $6(75-100 \%)$, de tal forma que 60 ( $6 \times 10$ parcelas) es el valor máximo de una especie en un transecto y representa el 100\%. Para determinar la magnitud de los cambios en la diversidad, debida a los componentes espaciales y temporales, se empleó el análisis de diversidad con interacción (Alatalo y Alatalo 1977) a dos escalas: a) detallada, considerando el ciclo anual y cada parcela de cada transecto (gradiente de profundidad) y b) general, empleando el ciclo anual y cada transecto (ambiente). En el primer análisis (por parcela), los componentes fueron: 1) intra parcelas - intra épocas: correspondiente a la diversidad $\alpha$ (riqueza) presente en cada parcela en cada época; 2) intra épocas - inter parcelas: indicando cuanto se incrementa la diversidad de cada parcela por efecto de las épocas; 3 ) inter épocas - intra parcelas: mostrando cuán diferente es la composición de la comunidad entre las parcelas en una misma época; 4) Interacción: mide el efecto de la interacción épocas-parcelas sobre la riqueza de especies. En el segundo análisis (por transecto), los componentes no variaron, pero se consideró la riqueza en cada transecto en lugar de en cada parcela.

Se aplicó el índice geométrico de las distancias Euclídeas para examinar el grado de disimilitud en cada ambiente a lo largo del ciclo inundación-sequía. Se emplearon las abundancias mensuales de especies en cada transecto, para agrupar aquellas épocas o meses del año en las cuales la composición de especies fue similar. Con el fin de detectar discontinuidades en pequeñas unidades (meses del año en un mismo transecto) se emplearon uniones simples o "single-linkage" para fusionar los grupos en el cluster.

\section{RESULTADOS}

Cobertura. Sólo un transecto (laguna $\mathrm{La}$ Cochina) se encontró totalmente desprovisto de vegetación durante un largo periodo del año (7 meses). El resto de los ambientes mantuvo una cubierta vegetal más o menos constante durante el año de estudio. Los ambientes con la cobertura menos variable fueron el estero Rabo de Iguana II (6.4\% en diciembre y $10 \%$ en agosto y septiembre) y la laguna Macolla de Caujaro (5.63\% en mayo y $10.2 \%$ en marzo).

Caños: la cobertura vegetal en el caño Macanillal evolucionó progresivamente a lo largo del año, asociada al régimen de inundación. A medida que avanzó la sequía y disminuyó el nivel hidrométrico, el porcentaje se redujo hasta su mínimo (3.2\%) en el mes más seco (abril). Igualmente en el caño Mucuritas, el mínimo (6.1\%) se alcanzó en sequía (abril) y el máximo (13.2\%) en aguas altas (julio).

Esteros: en Rabo de Iguana I, encontramos un patrón similar al de los caños. En los meses de aguas altas (julio, agosto y septiembre) el transecto estuvo más provisto de vegetación acuática que en el resto del año (13\%). El estero Rabo de Iguana II, fue el ambiente más homogéneo del conjunto, en cuanto a la cobertura vegetal. No hubo cambios drásticos durante el ciclo, alcanzándose el valor máximo en el mes de aguas altas (julio, 11.1\%).

Lagunas: la evolución de la cobertura vegetal fue similar en las cinco lagunas. Destaca Macolla de Caujaro como la más homogénea del conjunto, con mínimo en mayo (5.6\%) y máximos en febrero y marzo (10.1 y $10.2 \%$ ). En las lagunas restantes el porcentaje disminuyó desde el final de las lluvias hasta el mínimo en los meses más secos: marzo (El Boral II, 4.7\%), abril (Las Ventanas, 3.4\%) y mayo (El Boral I, 1.9\% y La Carmera, 3.6\%) y máximos durante las aguas altas julio (El Boral I, $13.2 \%$; La Carmera, $12.9 \%$ y Las Ventanas, 
13\%) y agosto (El Boral II, 12\%). En general la cobertura vegetal aumenta a mediados del año, en los meses de lluvia y es menor de enero a marzo (final de la sequía).

Frecuencia acumulada y riqueza de especies. La frecuencia acumulada de especies se obtuvo de los inventarios mensuales. Se registraron catorce especies en los catorce transectos durante el primer mes de muestreo (enero) y se alcanzó la riqueza máxima de 69 especies en el mes de octubre valor que se mantuvo constante hasta diciembre. La riqueza total de los transectos (69 sp.) (anexo 1), representó el $36 \%$ del inventario de especies de plantas acuáticas del Hato El Frío (Rial 2001). La riqueza mensual en todos los transectos, agrupados en caños, esteros y lagunas, se muestra en la figura
1 a-c. En general sigue la misma tendencia que la cobertura, aumento en entrada de lluvias y disminución al final de la sequía.

Caños: el transecto del caño Macanillal tiene un promedio de cuatro especies en los primeros meses del año (sequía), que aumenta hasta 13 en el mes de septiembre (final de las lluvias) manteniéndose con un promedio de 12 especies en la segunda mitad del año, correspondiente a las lluvias e inicio de la sequía. En el transecto del caño Mucuritas los meses de lluvia propiciaron los mayores valores de riqueza (15 sp. en junio).

Esteros: en estos ambientes los niveles del agua suelen ser poco variables. La riqueza total fue de 48 especies ( $67 \%$ del total), la más homogénea en relación a caños y lagunas. El final de la sequía (febrero) parece asociada a
A
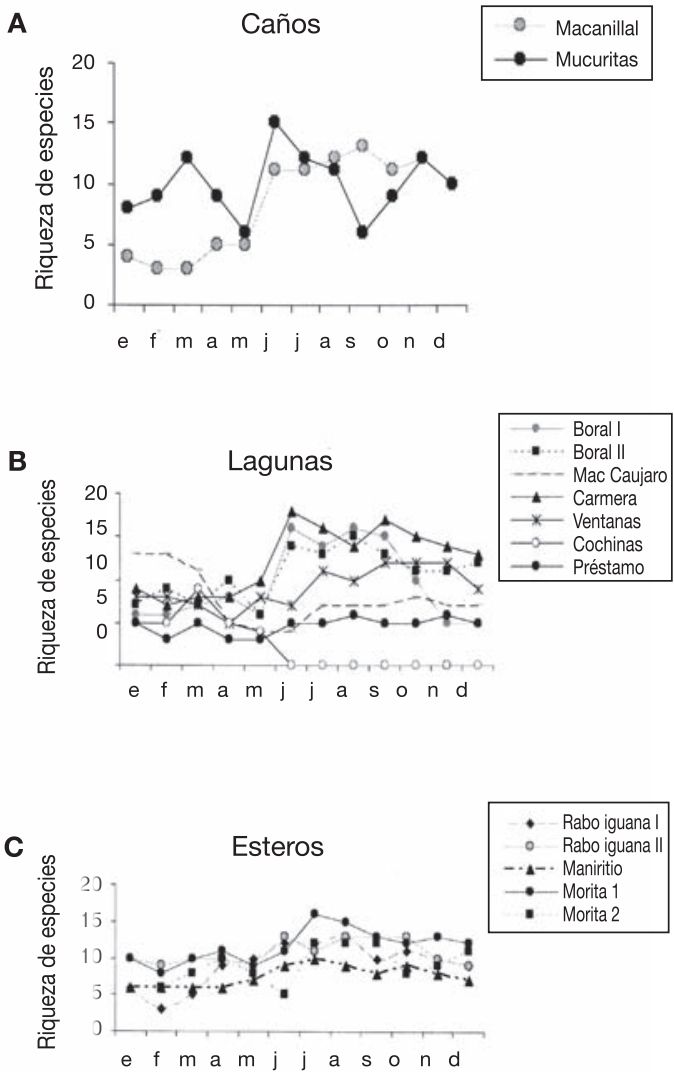

D

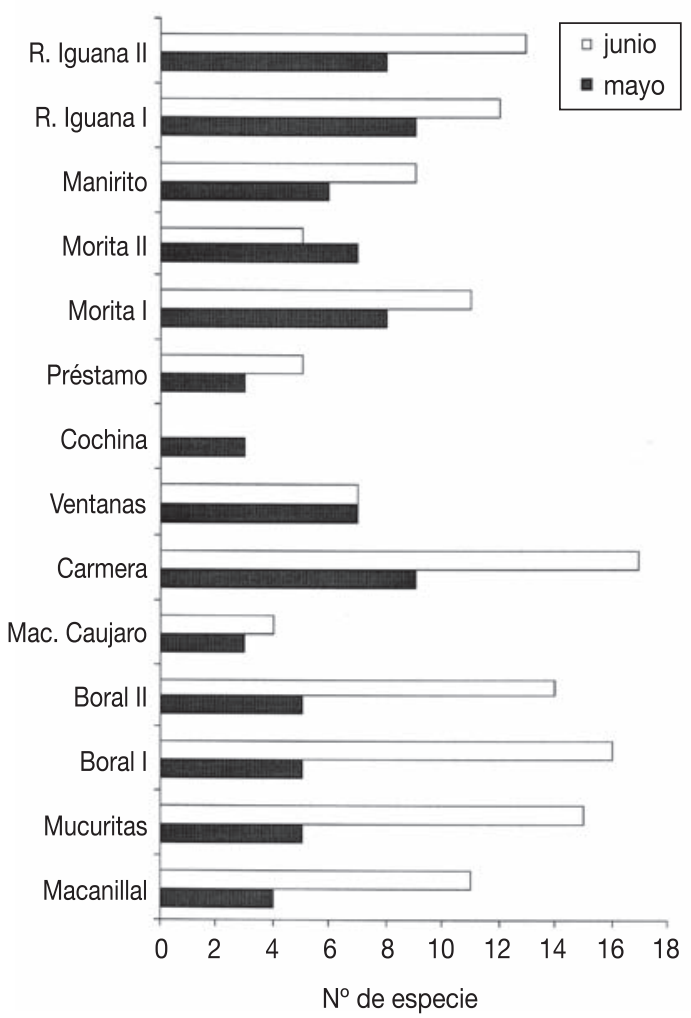

Fig. 1. Variación mensual de la riqueza de plantas acuáticas en los catorce transectos de estudio (A,B,C); especialmente en el periodo crítico: sequía-inicio de lluvia (D). 
la menor riqueza específica en los ambientes Rabo de Iguana I y II y Morita I, (3, 9 y 8 sp.) y en Manirito ( 6 sp. en abril). Excepcionalmente, la Morita II registró el menor número de especies al inicio de las lluvias (5 sp. en junio). El mayor número de especies se observó en todos los casos durante los meses de lluvia, entre junio y septiembre.

Lagunas: la riqueza total de las lagunas estudiadas fue de 55 especies, la mayor de todos los ambientes. En general fue menor en los meses iniciales del año, salvo en la laguna Macolla de Caujaro, en donde el comportamiento de la curva muestra la tendencia opuesta: mayor número de especies en sequía (13 sp. en enero y febrero), una drástica disminución al final de éste periodo (4 sp.) y un leve aumento al inicio de las lluvias (7 sp.) que se mantiene casi constante hasta el final del año. En la laguna La Carmera - la más rica de todas- el número de especies aumenta al inicio de las lluvias (18 sp. en junio) y disminuye levemente en aguas altas (14 sp.) manteniéndose el promedio hasta el final del año. Este comportamiento concomitante con el régimen de lluvia-sequía se observa también en las lagunas Boral I y II.

La intensidad de los cambios en la riqueza especifica de los ambientes durante el periodo crítico sequía-inicio de lluvias se muestra en la figura. 1d. El aumento del nivel del agua afectó diferencialmente a las comunidades de los diversos ambientes. En los cuerpos de agua temporales la respuesta fue inmediata, duplicándose el número de especies. Así ocurrió por ejemplo, en los caños - cuyos transectos fueron dispuestos en sectores represados o cercanos a diques- y lagunas de menor dimensión.

Variabilidad espacio-temporal de la riqueza. En el análisis de la diversidad con interacción (Alatalo y Alatalo 1977) en el que consideramos la estacionalidad y los transectos (tipo de ambiente), obtuvimos que la variable tipo de ambiente causa la mayor fuente de variación, incidiéndo sobre la riqueza en mayor grado (30.8) que la estacionalidad (14.1). El análisis más detallado que considera la estacionalidad y cada parcela del transecto (gradiente), muestra que las fluctuaciones hidrométricas, debidas al régimen biestacional, ejercen mayor influencia que la posición física dentro del gradiente de profundidad del transecto. El análisis determinó que once de los catorce transectos están más influenciados por el componente temporal, mientras que solo en cuatro ambientes (dos esteros y dos lagunas) la riqueza depende mayormente del gradiente de profundidad y del espacio físico que ocupa la parcela. En once casos la interacción fue negativa, indicando que el efecto conjunto de ambos componentes podría limitar la presencia de ciertas especies, es decir reducir la riqueza de especies. En el cuadro 2 se resume la contribución de cada fuente de variación en este análisis detallado (gradiente y estacionalidad).

Variabilidad espacio-temporal de la abundancia. La abundancia de las especies durante los doce meses del año en cada transecto, parece estar influenciada tanto por el ciclo inundación-sequía, como por la condición temporal o permanente del agua en cada ambiente. Los meses de abril, junio y julio (máxima sequía e inicio de lluvias) fueron los más disímiles en siete de los 14 transectos. En el resto de los casos, se generaron dos grandes grupos diferenciados justamente en los meses de lluvia (junio a agosto). La separación general de grupos se atribuye a la influencia de la inundación sobre la abundancia de las especies en cada ambiente. Así, en algunos casos (laguna Las Ventanas), el grupo julio y agosto (pico de lluvias) se diferenció del resto (Fig. 2), mientras que en el Caño Mucuritas los grupos más amplios muestran la diferencia paulatina que ocurre entre la sequía (diciembre a junio) y la inundación (julio-noviembre). La laguna La Carmera mostró pequeños grupos formados cada dos o tres meses, lo que indica pequeñas diferencias que solo son más evidentes en los meses de máxima sequía (abril-mayo). 
CUADRO 2

Análisis de la diversidad con interacción (Alatalo y Alatalo 1977)

empleando los componentes estacionalidad y parcelas (gradiente)

\begin{tabular}{|c|c|c|c|c|}
\hline & $\begin{array}{l}\text { Intra parcela- } \\
\text { Intra mes }\end{array}$ & $\begin{array}{l}\text { Inter mes- } \\
\text { Intra parcela }\end{array}$ & $\begin{array}{l}\text { Intra mes- } \\
\text { Inter parcela }\end{array}$ & $\begin{array}{l}\text { Interacción } \\
\text { mes-parcela }\end{array}$ \\
\hline $\mathrm{BI} \mathrm{N}^{\mathrm{o}} \mathrm{sp}$ & 3 & 9 & 7 & -0.5 \\
\hline$\%$ & 17 & 50 & 38 & 3 \\
\hline $\mathrm{B} 2 \mathrm{~N}^{\mathrm{o}} \mathrm{sp}$ & 5 & 14.1 & 5.8 & -3.3 \\
\hline$\%$ & 23 & 65 & 27 & 15 \\
\hline RI1 $N^{o} s p$ & 2.43 & 8.1 & 6.7 & -2.87 \\
\hline$\%$ & 17 & 57 & 43 & 20 \\
\hline RI2 $N^{o} s p$ & 4.7 & 8.4 & 6.1 & -2.4 \\
\hline$\%$ & 28 & 50 & 36 & 14 \\
\hline Man $\mathrm{N}^{\mathrm{o}} \mathrm{sp}$ & 3.4 & 4.2 & 4.2 & 3.4 \\
\hline$\%$ & 40 & 50 & 50 & 40 \\
\hline Mo1 $\mathrm{N}^{\mathrm{o}} \mathrm{sp}$ & 4.6 & 8.1 & 7.1 & -3.6 \\
\hline$\%$ & 29 & 51 & 45 & 23 \\
\hline $\mathrm{Mo} 2 \mathrm{~N}^{\mathrm{o}} \mathrm{sp}$ & 3.4 & 6.1 & 6.8 & -2.7 \\
\hline$\%$ & 25 & 45 & 50 & 20 \\
\hline $\mathrm{MC} \mathrm{N}^{\mathrm{o}} \mathrm{sp}$ & 4.1 & 7.3 & 3.7 & -0.5 \\
\hline$\%$ & 28 & 50 & 25 & 3 \\
\hline Mac $N^{o} \mathrm{sp}$ & 3.98 & 8.7 & 4.4 & -0.32 \\
\hline$\%$ & 24 & 52 & 26 & 2 \\
\hline Muc $\mathrm{N}^{\mathrm{o}} \mathrm{sp}$ & 5.1 & 11.24 & 4.9 & 1.24 \\
\hline$\%$ & 26 & 57 & 25 & 6 \\
\hline Pre $N^{o} s p$ & 1.53 & 2.78 & 3.1 & 1.21 \\
\hline$\%$ & 25 & 45 & 50 & 20 \\
\hline $\mathrm{Ch} \mathrm{N}^{\mathrm{o}} \mathrm{sp}$ & 0.9 & 6.4 & 1.4 & -4.1 \\
\hline$\%$ & 20 & 139 & 30 & 89 \\
\hline Ven $N^{o} \mathrm{sp}$ & 4.3 & 8.9 & 11 & -2.2 \\
\hline$\%$ & 20 & 40 & 50 & 10 \\
\hline Car $N^{o} \mathrm{sp}$ & 5.5 & 14.6 & 6.9 & -2.2 \\
\hline$\%$ & 22 & 59 & 28 & 9 \\
\hline
\end{tabular}

\section{DISCUSIÓN}

Los resultados obtenidos demuestran la influencia del régimen de inundación sobre la variación espacio temporal de la riqueza y abundancia de las especies de plantas acuáticas. En el área de estudio ambos parámetros aumentaron durante las lluvias y disminuyeron durante la sequía, siendo críticos los periodos de entrada de aguas y aguas altas. Investigadores con gran conocimiento de los sistemas inundables tropicales tales como Junk (1984), Neiff (1986) y Gopal (1990) entre otros, coinciden en señalar que las fluctuaciones hídricas ocasionan 


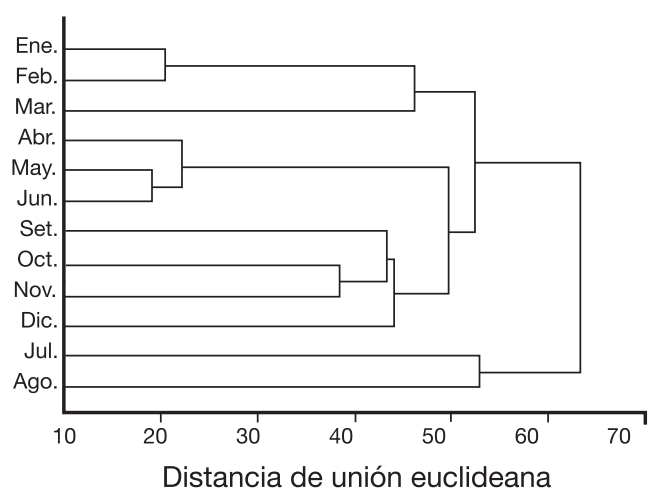

Fig. 2. Dendrograma de disimilitud de las comunidades vegetales acuáticas (abundancia) durante el ciclo hidrológico anual en la laguna Las Ventanas.

cambios drásticos sobre la diversidad y abundancia de las especies. Zutchi y Gopal (1990) destacan la influencia de la profundidad del agua y la periodicidad de la inundación sobre la composición de las comunidades vegetales que habitan en cuerpos de agua similares.

La singularidad de cada cuerpo de agua del Llano inundable, puede favorecer o limitar el establecimiento de determinadas comunidades vegetales dado que las características físicas de cada caño, laguna o estero, determinan distintos grados de afectación por las lluvias e inundación consecuente. El nivel de anegamiento de cada ambiente, depende de la capacidad de retención de agua de la cubeta, condición se relaciona con las dimensiones físicas, topográficas, morfológicas y con las características del suelo, en especial con el grado de permeabilidad (contenido de arcillas en su estructura). Algunos estudios muestran que pequeñas diferencias de elevación $(<0,5 \mathrm{~m}$ ) (van der Valk y Davies 1979), y consecuentemente de inundación, pueden generar mosaicos espaciales de diferentes tipos de hábitat que soportan a su vez diversas comunidades de plantas. Así pues, el régimen de lluvias ocasiona niveles máximos de inundación que pueden estar desfasados en los diferentes ambientes (Rial 2000). En consecuencia, los atributos y variables de las comunidades vegetales responden de modo particular a la inundación a pesar de que la tendencia general coincida con las fluctuaciones del agua en lluvia y sequía. Al respecto, tendremos presente que más que la comparación entre ambientes, la disposición de los transectos en el área de transición agua-tierra, permite establecer las semejanzas y diferencias entre las zonas más dinámicas del humedal. La cobertura (abundancia) vegetal de un estero en su zona de transición, es menor y a la vez más diversa que en el propio espejo de agua, ya que las zonas más profundas suelen ser menos susceptibles a las fluctuaciones hidrométricas y por ende, más estables.

La riqueza de especies también responde positivamente al aumento en el nivel del agua, especialmente al cambio drástico de condiciones durante la transición sequía-lluvia. Los ambientes de laguna fueron los más variados en términos de la composición de especies, seguidos de los esteros y los caños. La mayor complejidad del paisaje se observó durante el periodo de aguas altas en los ambientes de laguna. Las especies dominantes en cada ambiente (1-3 sp.) persistieron durante todo el ciclo. En general el conjunto de especie subordinadas se diferenció en dos grupos: uno que desapareció al secarse el suelo y otro que apenas sobrevivió un breve tiempo del ciclo (1 - 3 meses). De la condición temporal o permanente de los ambientes, se derivan diferencias en la composición y riqueza de las comunidades. Así, los transectos considerados temporales por cuanto se secan una parte del año, sufrieron un incremento en más del $50 \%$ de su riqueza específica durante el breve periodo de transición sequía-lluvias, es decir entre mayo y junio del año 1997. La entrada de las lluvias produjo un cambio inmediato en estas áreas, que en mayo mostraban suelos secos y en junio estaban saturadas de agua bajo una lámina de hasta medio metro de profundidad. Todos estos transectos tuvieron en común un gradiente de profundidad de escasa pendiente, originado con las primeras lluvias, con encharcamientos en la orilla y seguido de un suave desnivel hasta los $50 \mathrm{~cm}$ de profundidad en la parcela más 
distante (a los 10 metros). De esta manera, la mayor proporción de nuevas especies coloniza las zonas someras a lo largo de dicho gradiente. En la laguna Macolla de Caujaro por ejemplo, el fenómeno de transición fue más brusco, de la desecación total se pasó a una inundación con escaso gradiente -debido a la morfología de la orilla- que no favoreció la colonización de nuevas especies, salvo de Oxycaryum cubense. Por el contrario, permanecieron las especies ya presentes (Eichhornia crassipes Eleocharis intersticta e Hymenachne amplexicaulis) hecho que nos recuerda la condición oportunista de muchas comunidades acuáticas señalada por Zutchi y Gopal (1990). También se esperaría una mayor riqueza en los suelos de la zona de transición, donde las plantas se descomponen en cada turno de inundación y sequía (Walker et al. 1999) y en donde los beneficios de una mayor cantidad de nutrientes pueden ser aprovechados por las especies que allí se establezcan.

El análisis de la diversidad con interacción según el método de Alatalo y Alatalo (1977), muestra dos aspectos importantes: 1) el efecto temporal o estacional sobre la riqueza de especies ; 2) el efecto espacial, bien en términos del tipo de ambiente o espacio físico que ocupa dentro del humedal, o bien en términos del gradiente de profundidad o espacio físico que ocupa en el gradiente del transecto. Al mencionar el efecto temporal, nos referimos al ciclo de inundación-sequía que sucede en un periodo de doce meses.

El primer caso, en el que se analizan todos los transectos o ambientes a lo largo del año, pone en evidencia la contribución de las características del ambiente a la diversidad entendida como riqueza de especies. Efectivamente, esta diversidad puede ser mayor en las lagunas que en los caños, como hemos mostrado anteriormente. De muchas formas parece el medio más adecuado para el establecimiento de las especies de plantas acuáticas: la escasa turbulencia del agua, la aparente mayor disponibilidad de nutrientes por efectos de sedimentación y la morfología de las orillas, son factores intrínsecos del ambiente que favorecen la diversidad de las comunidades vegetales que las habitan. En ese primer análisis en el que se agrupan ambientes y meses, la contribución del ambiente supera así, al de la estacionalidad. Sin embargo, al afinar los términos, es decir, al sustituir los ambientes por microhábitat o parcelas, comprobamos el efecto de la inundación y la sequía sobre la riqueza de las comunidades y concluimos que su contribución es mayor que la del espacio físico que ocupan las especies en el gradiente. Consideramos entonces el hecho de que en cada cuerpo de agua, las parcelas -que se sitúan en microhábitat consecutivos en el gradiente de profundidad- están sometidas a distintos niveles de inundación (debido a sus características físicas), de tal forma que no es la posición espacial lo que determina una mayor riqueza específica, sino la condición de humedad. De este modo, la disponibilidad de agua en lluvias y sequía, contribuye en mayor grado a incrementar este valor, entendido aquí como diversidad. Con respecto a estos dos niveles de análisis y sus resultados, parecen apropiadas las siguientes observaciones. Las pequeñas diferencias topográficas en los ambientes, generan mosaicos de hábitat, microhábitat de comunidades vegetales diferentes (van der Valk y Davies 1979) y cualquier fluctuación temporal será impuesta a éstos patrones espaciales (Greening y Gerritsen 1987). Lo anterior podría apoyar la recomendación de Neiff (1986) en cuanto a caracterizar el paisaje y delimitar sus unidades considerando su variabilidad temporal.

Pielou (1984) mostró que a largo plazo, la estabilidad del ecosistema y la comunidad es dependiente de la estabilidad del ambiente. Pero los humedales no son precisamente ambientes estables. Aún cuando los cambios puedan ser predecibles, siguen siendo cambios en toda la extensión de la palabra. Estos cambios o perturbaciones pueden favorecer la diversidad (Connel 1978), y pueden considerarse a la vez un factor que favorece la adaptación y evolución de las especies (Tsimilli-Michael et al. 1996). Neiff (2001) afirma que los humedales influenciados por el pulso de inundación fluvial, poseen mayor riqueza de especies o diversidad alfa.

De los resultados obtenidos del método de agrupación concluimos una vez más que la 
estacionalidad influye efectivamente sobre las comunidades de plantas acuáticas en los distintos ambientes. En general, los meses de lluvia crearon la separación de grupos, de forma que la abundancia de especies mostró mayores diferencias en las distintas épocas de transición sequía-inundación de cada ambiente. En cada caso, las comunidades presentes a lo largo del año, se diferenciaron entre sí en grados distintos y esto puede tener causas variadas y de diversa índole: fisicoquímicas, morfológicas, hidrológicas, ecológicas, las cuales consideraremos en estudios posteriores. Por ejemplo, la laguna con la mayor riqueza de especies (La Carmera) mostró pequeños grupos cada dos o tres meses, lo que señala pequeñas diferencias que solo son más evidentes durante los meses de máxima sequía. De forma más drástica, en la laguna las Ventanas la diferencia entre los meses de pico de lluvias (julio y agosto) muestran una comunidad marcadamente distinta a la del resto del año. Todo esto puede ser observado fácilmente en el campo, ya que algunos ambientes cambian más drásticamente que otros y es sencillo hallar cierta relación con el grado y la forma en que la inundación ocurre.

En conclusión, observamos que en este humedal de los Llanos de Apure las diferencias temporales dentro de una comunidad de plantas acuáticas, son superiores y solo en algunos casos equiparables, a las diferencias espaciales con otras comunidades, es decir en otro tipo de ambiente acuático, tanto desde el punto de vista cualitativo como cuantitativo (composición y abundancia).

\section{AGRADECIMIENTOS}

A la Familia Maldonado e INVEGA C.A. por el apoyo logístico. C.A. Lasso, J. Castroviejo, Asociación Amigos de Doñana, L. Bulla, A. Aguirre y guías de la Estación Biológica El Frío.

\section{RESUMEN}

A fin de describir la variabilidad espacio temporal de las comunidades de plantas acuáticas en un humedal de los Llanos de Venezuela, se estudiaron su riqueza y abundancia, en 14 hábitat, durante un ciclo anual. En los transectos de estudio se identificó un total de 69 especies. Según el análisis de diversidad con interacción de Alatalo y Alatalo el aspecto temporal, es decir el régimen anual de lluvia-sequía y la consecuente variación del nivel del agua, es el factor que ejerce mayor influencia sobre la riqueza de especies, en relación al aspecto espacial, o tipo de hábitat. Según el índice geométrico de las distancias euclídeas, se concluye igualmente que la variación en la abundancia de especies se debe más a las fluctuaciones hidrométricas (estacionalidad) que al hábitat considerado.

\section{REFERENCIAS}

Alatalo, R. \& R. Alatalo. 1977. Components of diversity: multivariate analysis with interaction. Ecology 58: 900-906.

Beck, S.G. 1984. Comunidades vegetales de las sabanas inundables del NE de Bolivia. Phytocoenología 12: 321-350.

Braun-Blanquet, J. 1979. Fitosociología. H. Blume, Madrid. $820 \mathrm{p}$.

Connel, J.H. 1978. Diversity in tropical rain forest and coral reefs. Science 199: 1302-1310.

Colonnello, G. 1995. La vegetación acuática del Delta del Río Orinoco (Venezuela). Composición florística y aspectos ecológicos. Mem. Soc. Cienc. Nat. La Salle. 55(144): 3-34.

Gantes, P.H. \& N.M. Tur. 1993. Variación temporal de la vegetación en un arrollo de llanura. Rev. Brasil. Biol. 55: 259-266.

Gopal, B. \& K.P Sharma. 1990. Ecology of plant populations I: Growth. p. 79-106. In B. Gopal (ed.). Ecology and management of aquatic vegetation in the Indian subcontinent. Kluwer Academic, Dordrecht, Holanda.

Gordon, E. 2000. Dinámica de la vegetación y del banco de semillas en un humedal herbáceo lacustrino (Venezuela). Rev. Biol. Trop. 48: 23-42. 
Greening, H.S. \& J. Gerritsen. 1987. Changes in macrophytes community structure following drought in the Okefenokee swamp, Georgia, USA. Aquatic Bot. 28: 113-128.

Junk, W.J. 1984. Aquatic plants of the Amazon system. p. 319-337. In H. Sioli (ed.). The Amazon, limnology and landscape ecology of a mighty tropical river and its basin. Dr. W. Junk, Dordrecht, Holanda.

Junk, W.J. 1993. Wetlands of tropical South America. p. 679-739. In D.F. Whingham et al. (ed.). Wetlands of the world. Vol. 1 Inventory, Ecology and Management. Kluwer Academic, Dordrecht, Holanda.

Lasso, C. 2000. Peces de los Llanos de Venezuela: Guía Bioecológica de los Peces de la Estación Biológica El Frío y Caño Guaritico, Estado Apure. Publicaciones del Comité Español del Programa MaB y de la Red IberoMaB de la Unesco, $\mathrm{N}^{\circ} 3$ (en prensa)

Neiff, J.J. 1978. Fluctuaciones de la vegetación acuática en ambientes del valle de inundación del Paraná medio. Physis 38: 41-53.

Neiff, J.J. 1986. Aquatic plants of the Paraná system. p. 557571. In B.R. Davies \& K.F. Waker (eds.). The ecology river system. Dr. W. Junk, Dordrecht, Holanda.

Neiff, J.J. 2001. Diversity in some tropical wetland systems of south America. p. 157-186. In B. Gopal, W.J. Junk \& J.A. Davies (eds.). Biodiversity in wetlands: assessment, function and conservation. Vol. 2 Backhuys, Leiden, Holanda.

Margalef, R. 1974. Ecología. Omega, Barcelona, España.

Mereles, M.F. \& N. López de Kochalca. 1992. Humedales del Paraguay: breve reseña de su vegetación. Amazoniana 12(2): 305-316.
Pielou, E.C. 1984. The Interpretation of Ecological Data. Wiley Interscience, Nueva York. 263 p.

Rial, B.A. 2000. Aspectos cualitativos de la zonación y estratificación de comunidades de plantas acuáticas en un humedal de los Llanos de Venezuela. Mem. Fundación La Salle Cienc. Nat. 60(153): 69-85.

Rial, B.A. 2001. Plantas acuáticas de los Llanos inundables del Orinoco. Estado Apure, Venezuela: contribución taxonómica y ecológica. Tesis de doctorado, Universidad de Sevilla, Sevilla, España. 552 p.

Tsimilli-Michael, M., G.H. Kruger \& R.J. Strasser. 1996. About the perpetual state changes in plants approaching harmony with their environment. Archs. Sci. Genève. 49(2): 173-203.

Valk, van der A.G. \& C.B. Davies. 1979. A reconstruction of the recent vegetational history of a prairie marsh, Eagle Lake, Iowa, from its seed bank. Aquatic Bot. 6: 29-51.

Walker, I., R. Miyai \& M.D.A. de Melo. 1999. Observations on aquatic macrophyte dynamics in the reservoir of the Balbina Hydroelectric powerplant, Amazons state, Brazil. Acta Amazonica. 282: 243-265.

Werkhoven, M.C. \& G.M. Peeters. 1993. Aquatic macrophytes. p. 99-112. In P.E Quboter (ed.) Freshwater ecosystems of Suriname. Kluwer Academic, Dordrecht, Holanda.

Zutshi, D.P. \& B. Gopal.1990. Structure and dynamics of plant communities. p. 127-146. In B. Gopal (ed.). Ecology and management of aquatic vegetation in the indian subcontinent. Kluwer Academic, Dordrecht, Holanda. 
ANEXO 1

Lista de especies identificadas en los catorce transectos de estudio. Hato El Frío, Llanos inundables de Apure, Venezuela

Aeschinomene evenia Wright

Caperonia palustris (L.) St. Hill

Ceratopteris pteridoides (Hook) Hieron

Cynodon dactylon L. Pers.

Cyperus celluloso-reticulatus Boeck

Cyperus ochraceus Vahl.

Echinochloa colona (L.) Link.

Echinodorus grandiflorus

(Cham. Et Schl.) Mich

Eclipta prostrata (L.) L.

Eichhornia azurea (Sw.) Kunth

Eichhornia crassipes (Mart.) Solms

Eichhornia heterosperma Alexander

Eleocharis interstincta (Vahl) R \& S

Eleocharis mutata (L.) Roem \& Schult

Eleocharis sp.

Heliotropium procumbens Mill.

Hydrocleys parviflora Seub.

Hydrolea spinosa $\mathrm{L}$.

Hymenachne amplexicaulis (Rudge) Nees

Ipomoea asarifolia (Desv.)

Roem. \& Shult

Ipomoea carnea Jacq.

Justicia laevilinguis (Nees) Landau

Landoltia punctata (G. Mey)

Les y Crawford

Leersia hexandra Swartz

Limnobium laevigatum (H\&B ex. Willd)

Heine

Ludwigia erecta (L. ) Hara

Ludwigia helmintorrhiza (Mart.) Hara

Ludwigia inclinata (L.f. ) Raven

Ludwigia octovalvis (Jacq.) Raven

Ludwigia peploides (H.B.K.) Raven

Ludwigia sedoides ( H.\& B.) Hara

Luziola subintegra Swallen

Marsilea polycarpa Hook \& Grew

Melochia manducata Wright

Melochia sp.
CAR 17583

CAR 17510

CAR 18608

CAR 17625

CAR 17219

CAR 17231

CAR 17634

CAR 17348

CAR 17212

CAR 17422

CAR 17419

CAR 17425

CAR 17758

CAR 17750

CAR 17329

CAR 17398

CAR 17520

CAR 17332

CAR 17213

CAR 17502

CAR 17457

CAR 18599

CAR 17665

CAR 17386

CAR 17565

CAR 17567

CAR 17571

CAR 17572

CAR 17578

CAR 17580

CAR 17690

CAR 18618

CAR 17254

CAR 17619
Melothria trilobata Cong.

Mikania congesta DC.

Mimosa pigra L.

Najas arguta H.B.K.

Neptunia oleraceae Lour.

Nymphaea novogranatensis Wiersema

Nymphoides humboldtiana (Kunth) Kuntze

Oryza rufipogon Griff

Oxycaryum cubense (Poepp. \& Kunth)

Lye

Pacourina edulis Aublet.

Panicum elephantipes Nees

Paratheria prostrata Nees

Paspalum repens Berg.

Pistia stratiotes $\mathrm{L}$.

Polygonum punctatum Ell.

Pontederia subovata (Seub.) Lowden

Reimarochloa acuta (Fluge) Hitch

Sagittaria guayanensis H.B.K.

Salvinia auriculata Aubl.

Salvinia sprucei Kuhn

Senna aculeata (Bentham)

Irwin \& Barneby

Sesbania exasperata H.B.K.

Solanum hirtum Vahl

Spermacoce scabiosoides

(Cham. \& Shul) Kuntze

Spermacoce verticillata $\mathrm{L}$.

Spilanthes ocymifolia (Lam.)

A.H. Moore

Thalia geniculata $\mathrm{L}$.

Trichospira verticillata (L.) Blake

Urena sinuata $\mathrm{L}$.

Urochloa brizantha (Horchst. ex. Rich.)

R. Webster

Utricularia hydrocarpa Vahl.

Utricularia inflata Walter

Utricularia foliosa $\mathrm{L}$.

Utricularia spp.
CAR 17534

CAR 17507

CAR 17479

CAR 17553

CAR 17414

CAR 18613

CAR 17253

CAR 17548

CAR 17691

CAR 17772

CAR 17234

CAR 17702

CAR 17708

CAR 17718

CAR 17375

CAR 17342

CAR 17442

CAR 17719

CAR 17360

CAR 18611

CAR 18609

CAR 17491

CAR 17584

CAR 17601

CAR 17605

CAR 17236

CAR 17410

CAR 17485

CAR 17454

CAR 17728

CAR 17503 
